\title{
Structure of an atypical homodimeric actin capping protein from the malaria parasite
}

\author{
Ábris Ádám Bendes ${ }^{\mathrm{a}}$, Petri Kursula ${ }^{\mathrm{a}, \mathrm{b}}$, and Inari Kursula, ${ }^{\mathrm{a}, \mathrm{b}^{*}}$ \\ ${ }^{\mathrm{a}}$ Biocenter Oulu and Faculty of Biochemistry and Molecular Medicine, University of Oulu, P.O. \\ Box 5400, 90014, Oulu, Finland \\ ${ }^{\mathrm{b}}$ Department of Biomedicine, University of Bergen, Jonas Lies vei 91, 5009, Bergen, Norway \\ *Corresponding author, e-mail address: inari.kursula@uib.no
}

Keywords: actin capping protein, homodimer, glideosome, malaria, Plasmodium

\begin{abstract}
Actin capping proteins (CPs) are essential regulators of actin dynamics in all eukaryotes. Their structure and function have been extensively characterized in higher eukaryotes but their role and mechanism of action in apicomplexan parasites remain enigmatic. Here, we present a crystal structure of a unique homodimeric $\mathrm{CP}$ from the rodent malaria parasite Plasmodium berghei. In addition, we compare homo- and heterodimeric arrangements of $P$. berghei $\mathrm{CPs}(\mathrm{PbCPs})$ in solution. We complement our findings by describing the regulatory effects of $P b C P s$ on heterologous skeletal muscle $\alpha$-actin as well as parasite actin. Comprehensive kinetic and steadystate measurements show atypical regulation of actin dynamics; $\mathrm{PbCPs}$ facilitate rapid turnover of parasite actin I without affecting the apparent critical concentration. Possibly to rescue actin filament capping in life cycle stages where the $\mathrm{CP} \beta$-subunit is downregulated, homo- and heterodimeric $\mathrm{PbCPs}$ show redundant effects in vitro. However, our data suggest that homodimers may in addition influence actin kinetics by recruiting lateral actin dimers. This unusual function could arise from the absence of a $\beta$-subunit, as the asymmetric $\mathrm{PbCP}$ homodimer lacks the structural elements essential for canonical barbed end interactions, suggesting a novel $\mathrm{CP}$ binding mode. These findings facilitate further studies aimed at elucidating the precise actin filament capping mechanism in Plasmodium and the eligibility of PbCPs as drug targets against malaria.
\end{abstract}




\section{Significance statement}

Malaria parasites of the genus Plasmodium display a unique form of gliding motility, which depends on an unconventional actomyosin motor. Actin capping proteins (CPs) play a major role in regulating parasite motility. Here, we describe a unique Plasmodium berghei $\mathrm{CP}(\mathrm{PbCP})$ system, behaving contradictory to canonical heterodimeric CPs, more suited to regulate the fast dynamics of the parasite actin system. We present the crystal structure of a distinctive homodimeric form of $\mathrm{PbCP}$ and extensive biochemical data, describing the atypical behavior of each $\mathrm{PbCP}$ form. The $\mathrm{PbCP}$ homodimer displays capping even in the absence of canonical conserved structural elements, suggesting a novel actin-CP interaction mode. These distinct structural properties could provide opportunities for drug design against malaria.

\section{Author contributions}

I.K. and P.K. conceived the study. Á.Á.B. performed the experiments, analyzed the data, and drafted the manuscript. Á.Á.B., I.K. and P.K. solved and refined the crystal structure. Á.Á.B. and I.K. wrote the final manuscript and all authors approved the final version of the manuscript. 


\section{Introduction}

Actin is a major element of the eukaryotic cytoskeleton and essential for a spectrum of cellular functions, including cell motility, intracellular transport, endocytosis, and cytokinesis. The versatile behavior of actin-based systems requires tight spatial and temporal control that is conveyed by a vast number of regulatory proteins (1). Actin capping proteins (CPs) are heterodimeric proteins, ubiquitously present in eukaryotes (2). CPs play a crucial role in actin dynamics by binding with high affinity to the fast-growing barbed end of filamentous actin (F-actin) in a $\mathrm{Ca}^{2+}$ independent manner (3), limiting protomer exchange to the pointed end. CPs are present in various cytoskeletal structures, such as lamellipodial protrusions (4), dynactin (5), and in the sarcomere, linking microfilaments to the Z-disks (6). Most vertebrates encode multiple isoforms of CP subunits with diverse functions (2). CPs are essential for human and zebrafish morphogenesis (7) and they belong to the core set of proteins needed to reconstitute actin-based motility in vitro (8). The average cytosolic concentration of $\mathrm{CP}$ in eukaryotes is in the range of $0.5-1.5 \mu \mathrm{M}(9,10)$, which, considering the high affinity and 1:1 stoichiometry of CP towards actin filaments, leads to a high number of constantly capped barbed ends in vivo (10). However, formation of free barbed ends is essential for rapid actin network assembly $(11,12)$, and control of CP expression levels is required for optimal actin-based cellular functions $(8,9)$. Thus, the mechanism of capping/uncapping is modulated by various external factors. Steric and allosteric regulators of CPs include polyphosphoinositides (PIPs), V-1/myotrophin, and CARMIL proteins, while indirect barbed end competitors include formins and ENA/VASP proteins (2).

CPs are comprised of two subunits, $\mathrm{CP} \alpha$ and $\mathrm{CP} \beta$, each with an approximate molecular weight of 32-36 kDa (2). In most eukaryotes, the individual subunits are conserved, although the sequence identity between the subunits is typically low (2). Despite the sequence divergence, the subunits share a strikingly similar fold, resulting in a unique quaternary structure where the two subunits take up a compact arrangement with a pseudo two-fold symmetry (13). All currently available structures of heterodimeric CPs resemble the characteristic shape of a stipitate mushroom $(5,13,14)$. Three N-terminal antiparallel helices form the "stalk" domain flanked by a characteristic $\beta$-stranded "globule" domain of each monomer. The "cap" is comprised of a well-ordered arrangement of two 
5-stranded antiparallel $\beta$-sheets, crested by a backbone of four helices formed conjunctly by the subunits. Emerging from the cap structure are the C-terminal "tentacles" of each subunit (termed $\alpha$ and $\beta$-tentacle, respectively) containing a longer flexible loop region and an amphipathic helix (13). According to the current view, CPs bind the terminal two actin protomers of a filament in a sequential mode that involves a coordinated interplay of a positively charged patch on the cap and both tentacle domains of the subunits $(15,16)$.

Alongside other members of the vast phylum of Apicomplexa, malaria parasites (Plasmodium spp.) use a special actomyosin motor for motility and host cell invasion (17). These parasites have two non-canonical actin isoforms and a modest set of 10-15 actin-binding proteins, lacking identifiable orthologues of the Arp2/3 complex, the majority of capping/severing/cross-linking proteins, or regulators like ENA/VASP proteins $(17,18)$. Contrary to the majority of eukaryotes, Plasmodium spp. encode only one isoform of each CP subunit (19). Metazoan CPs characterized to date nucleate polymerization, decrease elongation rate in preseeded systems, block dilution-induced depolymerization from barbed ends, and increase critical concentration to the level of the pointed end (2). Even though individual CP subunits and isoforms are expressed at different levels during different stages and cell types (20), CP subunits are largely insoluble and non-functional when expressed alone in vitro, indicating that they only exist as heterodimers (21). In Plasmodium, however, in addition to a heterodimeric CP $(19,22)$, a unique homodimer form of the $\alpha$-subunit exists and displays capping activity of homo- and heterologous actin filaments in vitro $(23,24)$. Here, we present the first crystal structure of a homodimeric CP from the rodent malaria parasite Plasmodium berghei $(P b \mathrm{CP} \alpha \alpha)$. The structure demonstrates critical differences compared to canonical heterodimeric CPs. We complement our structural findings with extensive biochemical characterization of $P b C P \alpha \alpha$ and the $P$. berghei CP heterodimer (PbCP $\alpha \beta)$, showing that the homoand heterodimers have distinct functions that differ from the canonical CP heterodimer (CapZ $\alpha \beta)$ and are specific to the parasite actin filaments. 


\section{Results and discussion}

\section{Plasmodium CPs form similar-shaped homo- and heterodimers in solution}

$P b C P \alpha \alpha$ without the $\alpha$-tentacle $\left(P b C P \alpha \alpha^{\Delta \mathrm{C} 20}\right)$ forms dimers with the canonical mushroom shape of CPs (24), but it was not clear whether the full-length $P b C P \alpha \alpha$ also could homodimerize in a similar manner. Here, we set out to investigate the quaternary arrangements of the full-length homo- and heterodimeric PbCPs as well. Using a combination of small-angle X-ray scattering (SAXS) and homology modeling based on related crystal structures, we show that, indeed, all three parasite CP versions (the truncated and full-length $P b C P \alpha \alpha$ and $P b C P \alpha \beta$ ) form similar pseudo-symmetric dimers in solution (Fig. 1). The SAXS data show folded, globular proteins with flexible parts, $P b C P \alpha \beta$ being somewhat more compact than the homodimers (Fig. 1A and B). Interparticle distances are similar for all PbCPs, with the heterodimer showing slight bimodality (Fig. 1C). Modeling into the SAXS data suggests that $P b C P \alpha \beta$ forms a tight structure, whereas the homodimers adopt a looser structure, which may arise from steric hindrances at the dimer interface (Fig. 1D). The orientation of a Plasmodium-specific insert of $P b C P \alpha(24)$ and the tentacle domains of both subunits cannot be reliably deduced from the SAXS data, indicating that they are disordered in solution, which is characteristic of the tentacles in canonical CPs as well $(13,14)$.

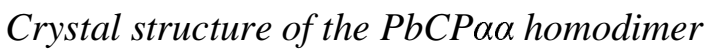

To gain further structural insight into the unusual homodimerization of the parasite $\mathrm{CP}$, we crystallized and determined the structure of the truncated $P b C P \alpha \alpha^{\Delta C 20}$ (Fig. 2A), as the full-length protein as well as the heterodimer have so far resisted crystallization. Extensive attempts to solve the structure using various molecular replacement approaches failed, pointing towards significant subunit- and domain-wise structural rearrangements in $\mathrm{PbCP} \alpha \alpha^{\Delta \mathrm{C} 20}$ compared to canonical CP heterodimers. We finally succeeded in solving the structure to $2.2 \AA$ resolution using singlewavelength anomalous diffraction of bromide derivatives (Table I). As expected from SAXS analysis from before (24) and in this work, the two $P b C P \alpha^{\Delta \mathrm{C} 20}$ subunits form a less compact structure than the canonical heterodimer (Fig. 2A), still closely resembling the established mushroom shape of metazoan CPs $(13,14)$, despite the low sequence conservation (23). Albeit being a homodimer, the structure is not completely symmetric. As predicted by homology modeling 
and SAXS data, significant structural rearrangements are required at the dimer interface to accommodate two identical subunits in the dimer (Fig. 2B). This results in a new type of noncanonical dimeric CP structure and a new example of rare asymmetric homodimers among proteins in general (25). Because of the asymmetry, we will here refer to chain A of the crystal structure as $P b C P \alpha_{1}{ }^{\Delta \mathrm{C} 20}$ and chain $\mathrm{B}$ as $P b C P \alpha_{2}{ }^{\Delta \mathrm{C} 20}$. While in canonical heterodimers the stalk domains of the subunits pivot relative to the core structure to accommodate denser packing, in $P b C P \alpha \alpha^{\Delta \mathrm{C} 20}$, the orientation is the same in both subunits, disrupting the compact structure (Fig. 2C). Further major structural deviations include longer helices of the stalk domains, a Plasmodium-specific insert between the globule and cap $\beta$-sheets (Ser135-Ala159), and a unique $\beta$-hairpin-like C-terminus (Leu268-Leu286) of the $P b C P \alpha_{1}{ }^{\Delta \mathrm{C} 20}$ H5 helix (Fig. 2).

Due to the reduced number of intersubunit and crystal contacts, $P b C P \alpha_{2}{ }^{\Delta \mathrm{C} 20}$ is more disordered, with a higher average B-factor than $P b C P \alpha_{1}{ }^{\Delta \mathrm{C} 20}$ (Suppl. Fig. S1 and Table I), similar to what has been described for CapZ $\alpha \beta$ (14). Consequently, the Plasmodium-specific insert (residues 134-155) is also not visible in $\mathrm{PbCP}_{2}{ }^{{ }_{\mathrm{C}} 20}$. In $P b \mathrm{CP} \alpha_{1}{ }^{\Delta \mathrm{C} 20}$, this loop protrudes from the structure, making space for the long helix $\mathrm{H} 5$ (residues 243-283) of $\mathrm{PbCPa}_{2}{ }^{\Delta \mathrm{C} 20}$, which would precede the $\alpha$-tentacle in the full-length protein. In $P b C P \alpha_{1}{ }^{\Delta \mathrm{C} 20}, \mathrm{H} 5$ is broken at residue 267 and turns back towards the same subunit in a $\beta$-hairpin-like structure, instead of extending into $P b C P \alpha_{1}{ }^{\Delta C 20}$. The base of the Plasmodium-specific loop seems to be stabilized by a partial disulfide bond between Cys158 and Cys179 in the $P b C P \alpha_{1}{ }^{\Delta \mathrm{C} 20}$ subunit but not in $P b C P \alpha_{2}{ }^{\Delta \mathrm{C} 20}$. The loose nature of the homodimer and the substantial structural differences in the $\mathrm{C}$-terminal region could result in a non-canonical binding mode to the highly dynamic Plasmodium actins $(26,27)$ and a distinct role for the homodimers as compared to the canonical or the parasite CP heterodimers $(19,23,24)$. In comparison to the sarcomeric [PDB ID: 1IZN (13)], cytoplasmic [PDB ID: 4AKR (14)], and dynactin bound [PDB ID: 6F1T (28)] CP isoforms, $P b C P \alpha \alpha^{\Delta C 20}$ has an increased surface area but significantly decreased dimer interface area (Suppl. Table SI), as is typical for asymmetric homodimers (25). Root-mean-square deviations (r.m.s.d.) calculated between these structures reveal further insights on a dimer, subunit, and domain level (Suppl. Table SII). Canonical heterodimers are much more similar to each other than to the Plasmodium homodimer (Suppl. Table SIIA), owing to their subunit arrangement. CP-related CATH domains (1.20.1290.20 and 2.40.160.80) are 
present in $\mathrm{PbCP} \alpha \alpha^{\Delta \mathrm{C} 20}$, manifested by a lower r.m.s.d. when individual subunits are compared. Both $P b C P a \alpha^{\Delta \mathrm{C} 20}$ subunits are more similar to canonical $\mathrm{CP} \alpha$, with $P b C P \alpha_{2}{ }^{\Delta \mathrm{C} 20}$ being slightly closer to CP $\beta$ (Suppl. Table SIIB). Given sequence limitations, this subunit could facilitate a more canonical heterodimer arrangement of the Plasmodium homodimer. Individual domains of $P b C P a \alpha^{\Delta C 20}$ fit well to canonical CP subunits suggesting the importance of individual structural elements in the capping function (Suppl. Table SIIC), despite sequence differences.

\section{PbCPs control actin polymerization in a non-canonic manner}

CPs typically block the barbed end, increasing its apparent critical concentration $\left(\mathrm{Cc}_{\text {app }}\right)$ to that of the pointed end's (2). PbCPs increase the supernatant fraction of PfActI in pelleting assays (24), which could either be explained by filament shortening or by limited depolymerization due to the increase of $\mathrm{Cc}_{\text {app. }}$. In dilution series of fluorescently labeled $P f A c t I$ filaments, none of the $P b C P s$ affect $\mathrm{Cc}_{\mathrm{app}}$, even at high concentrations (Fig. 3A). Furthermore, gelsolin, a major barbed end capper (1) absent from Plasmodium spp. (18), does not affect the $\mathrm{Cc}_{\mathrm{app}}$ of PfActI filaments (Fig. 3B) or PfActI depolymerization dynamics (27). None of the PbCPs increase the $\mathrm{Cc}_{\text {app }}$ of heterologous skeletal $\alpha$-actin either (Fig. 3C), despite them reportedly being able to modulate $\alpha$ actin (22) or non-muscle $\beta$-actin polymerization $(19,23)$. We hypothesize that $\mathrm{PbCPs}$ have a lower affinity to actin than canonical CPs, allowing actin subunit exchange, making them wobbly or leaky cappers (2), similar to formins (1).

The inability of $\mathrm{PbCPs}$ to increase $\mathrm{Cc}_{\mathrm{app}}$ raises a question about the presence of other actin regulatory properties. In higher eukaryotes, CPs nucleate filaments, abolishing the lag phase, but block subunit exchange at the barbed end (2), reducing the initial elongation velocity and steadystate filament mass. In a homologous system, contrary to expected, we found that $\mathrm{PbCPs}$ increase elongation velocity and considerably raise the steady-state level of F-actin mass (Fig. 4A). Their effect on nucleation is ambiguous due to the nature of PfActI polymerization curves (27). In preseeded assays, the filament mass is less affected (Fig. 4B). CapZ $\alpha \beta$ does not seem to modulate PfActI polymerization notably. On the contrary, both $\mathrm{PbCP}$ homodimers and the heterodimer behave similarly to typical CPs with $\alpha$-actin (Fig. 4C and D). These results suggest that $\mathrm{PbCP}$ homo- and heterodimers have similar, redundant functions, which would secure actin capping 
functions in the blood stages of the parasite where $P b C P \beta$ is phenotypically absent (19).

$P b C P a \alpha^{\Delta \mathrm{C} 20}$ displays a diminished, yet comparable capping effect, despite the absence of the essential tentacle domains $(15,16)$. The reduced importance of the tentacle domain in PbCPs has been suggested before (24). However, in vivo, the Plasmodium $\alpha$-tentacle seems to be indispensable (23). The major difference between homo- and heterodimers is seen in the initial filament mass in non-seeded systems (Fig. 4A and C). It seems that while PbCPs are able to cap F-actin, in the absence of preformed filaments, the homodimers may also be able to stabilize or sequester short, non-nucleating structures, perhaps lateral dimers (27). This behavior of the homodimers extends to $\alpha$-actin and seems to be independent of the presence of the $\alpha$-tentacle.

\section{Dilution-induced disassembly of filaments is enhanced in the presence of PbCPs}

PfActI filaments are highly dynamic $(26,27)$ with high disassociation rates at both ends (29). Upon dilution below their $\mathrm{Cc}_{\mathrm{app}}$, filaments decompose rapidly, faster than $\alpha$-actin (27). We measured the barbed end blocking efficiency of $\mathrm{PbCPs}$, by following the disassembly of fluorescently labeled PfActI and $\alpha$-actin filaments. PbCPs facilitate depolymerization of both PfActI (Fig. 5A) and $\alpha$ actin filaments (Fig. 5B). While CapZ $\alpha \beta$ behaved as expected with $\alpha$-actin, reducing the rate of depolymerization (2), it also increased the rate of PfActI depolymerization, suggesting that the effect results from an interplay between Plasmodium actins and CPs, not strictly from the latter. While the suggested propensity of PbCPs to sequester actin dimers agrees well with the data, we cannot exclude a moonlighting function of these proteins as filament severing proteins (1). Such behavior of CPs has been described in the presence of VASP proteins (30). PbCPs, however, in the absence of ENA/VASP homologs (18), might have evolved to have inherent severing capability. This would raise the question of redundancy among the limited Plasmodium actin regulators, as multiple severing proteins have been described $(17,27)$.

\section{Implications of an atypical $C P$}

In vivo, many actin binding proteins (ABPs) exist to fine-tune barbed end capping through wellcharacterized mechanisms (2). While the majority of these are absent from Plasmodium $(17,18)$, we cannot exclude the possibility of other, so far uncharacterized, regulators. Our search for V- 
1/myotrophin homologs and the CP-binding and uncapping motif among known Plasmodium transcripts or for the S100B interaction motif in Plasmodium CPs resulted in no clear hits. To date, in P. knowlesi, $\mathrm{PIP}_{2}$ is the only described direct CP regulator, with $\mathrm{HSC} 70$ mentioned as a binding partner, uninvolved in capping activity (22). Residues involved in canonical interaction with proteinaceous regulators are generally not conserved in Plasmodium (Suppl. Fig. S2 and S3), with an exception of several basic residues (K278 and $\mathrm{K} 282$ in $P b C P \alpha, \mathrm{R} 233$ in $P b C P \beta)$ involved in canonical F-actin interaction and $\mathrm{PIP}_{2}$ binding $(15,16,31)$. Based on our kinetic data, $\mathrm{PbCPs}$ seem to have at least an order of magnitude lower affinity towards PfActI filaments compared to CapZ $\alpha \beta$ and canonical actin. Left unregulated, CPs would constantly cap actin filaments, completely arresting cell motility (10). We cannot rule out that this innate down-regulation of $P b C P s$ could allow for the existence of free barbed ends in the absence of other regulators, allowing the parasite to function with only a minimal set of ABPs.

Given the discussed substantial differences of $\mathrm{PbCPs}$ compared to canonical CPs, in particular the absence of a $\beta$-subunit in certain stages, we hypothesize that the F-actin binding mode of homodimeric PbCPs may also be different. Canonical CPs bind the barbed end through an electrostatic interaction mediated largely by the basic triad on CapZ $\alpha \beta$ and an acidic patch on the last two actin protomers (15). To understand whether this binding mode can exist in Plasmodium, we used molecular docking to generate a model of a $P b C P \alpha \alpha^{\Delta \mathrm{C} 20}$ capped PfActI filament (Fig. 6A). Presence of Arp1 in the P. berghei genome (32) could implicate the existence of PbCP capped Arp1 filaments in Plasmodium, thus allowing the cryo-EM structure of CapZ $\alpha \beta$ capped Arp1 filament in Sus scrofa dynactin (28) to be used as basis for further modeling studies. Electrostatic potential surfaces of the barbed end (Fig. 6B) and CPs (Fig. 6C) reveal the absence of canonical charged interaction points only for Plasmodium (Suppl. Fig. S4), even though residues involved in forming these interaction surfaces are conserved (Suppl. Fig. S2, S3, and S5). In PfActI, flanking residues mask the apparent charge of the patch (Fig. 6B). Differences in electrostatic potential surfaces (33) could also play a large role in the divergent nucleation and polymerization properties of PfActI (27). Residues of the basic triad in $\mathrm{PbCP} \alpha \alpha^{\Delta \mathrm{C} 20}$ are more buried in the structure, not in close proximity to each other and the slight asymmetry of the homodimer does not compensate significantly for the loss of contributing residues of $P b C P \beta$. Possibly due to the absence of CARMIL proteins in 
Plasmodium, a positively charged canonical binding cavity is not present in $\mathrm{PbCP} \alpha^{\Delta \mathrm{C} 20}$ (Fig. 6C). In the CapZ $\alpha \beta$ heterodimer, the $\beta$-tentacle locks the complex by binding the hydrophobic pocket of the terminal actin subunit $(15,16)$. In our model, the Plasmodium-specific insert of $P b C P \alpha_{1}{ }^{\Delta C 20}$ is close to the expected position of the $\beta$-tentacle. This insert shares many possible, hydrophobic or electrostatic, interaction points with the $\beta$-tentacle (Fig. 6D), residues mainly conserved in Plasmodium (Suppl. Fig. S2), suggesting a role for the insert in barbed end binding in the absence of canonical interaction motifs. Mapping sequence conservation of Plasmodium CP $\alpha$ sequences on the structure of $\mathrm{PbCP} \alpha \alpha^{\Delta \mathrm{C} 20}$ reveals that core residues, especially the ones involved in the intersubunit surface are highly conserved, suggesting that other CP $\alpha \alpha$ homodimers could exist in Plasmodium spp. Canonical binding partner interaction points present in CapZ $\alpha \beta$ lack localized sequence conservation in $P b C P \alpha \alpha^{\Delta \mathrm{C} 20}$ (Fig. 6E and Suppl. Fig. S2).

The truncated $P b C P \alpha \alpha^{\Delta \mathrm{C} 20}$ binds actin in the absence of the essential structural elements (24), suggesting that canonical functions of the tentacle domains are compensated by other means. Possibilities are that both interaction sites undergo significant conformational changes upon binding, or a completely novel mechanism is involved. Follow-up mutagenesis studies aimed at the Plasmodium insert and the surface residues of the capping structure could shed light to this unique interaction.

\section{Concluding remarks}

CPs are essential regulators of the cytoskeleton and conserved among metazoans as heterodimeric proteins, which are important e.g. for lamellipodial protrusions at the leading edge of crawling cells (4). Apicomplexan parasites display fast gliding motility in the absence of changes in cell shape (34). It seems that due to different needs during different lifecycle stages, these parasites have evolved an additional unique homodimeric form of CP (24) as part of their limited repertoire of ABPs (17). In contrast to canonical CPs (2), PbCPs facilitate the rapid turnover of the dynamic Plasmodium actin filaments. Due to the atypical structural and biochemical properties, and a unique mode of binding to the barbed ends as well as their essential nature, Plasmodium CPs could prove as promising drug targets against malaria.

\section{Materials and methods}




\section{Protein expression and purification}

$P b C P \alpha \beta, P b C P \alpha \alpha, P b C P \alpha \alpha^{\Delta \mathrm{C} 20}$, CapZ $\alpha \beta, \alpha$-actin, and PfActI were prepared as described previously $(24,27)$. Human full-length gelsolin was purchased from Cytoskeleton (US).

\section{Model refinement using small-angle X-ray scattering (SAXS)}

SEC-SAXS data of $P b C P \alpha \alpha$ and $P b C P \alpha \beta$, at a respective concentration of 12 and $5.8 \mathrm{mg} / \mathrm{ml}$, were collected on the B21 beamline at Diamond Light Source (Didcot, UK). Data processing, ab initio DAMMIN (35) modeling, and visualization were carried out as previously described (24). Initial models of the homodimers were prepared by extending the crystal structure of $P b C P a \alpha^{\Delta \mathrm{C} 20}$ using SWISS-MODEL (36). The tentacle domain of $P b C P \alpha_{1}$ was modeled using EOM (37). A model of $P b C P \alpha \beta$ was assembled from the modeled $P b C P \alpha_{2}$ subunit and I-TASSER (38) modelled PbCP $\beta$ monomer using the structure of dynactin-bound S. scrofa CapZ $\alpha \beta$ [PDB ID: 6F1T (28)] as a template. The models were corrected against major steric clashes using Coot (39) and energy minimized using UCSF Chimera (40). The PbCP models were split into domains and refined with normal mode analysis using SREFLEX (41).

\section{Crystallization and structure solution}

$P b C P \alpha \alpha^{\Delta \mathrm{C} 20}$ was crystallized at $4^{\circ} \mathrm{C}$ using the vapor diffusion method. Crystals were grown from a 1:1 drop ratio of 10-15 mg/ml protein and precipitant [100 mM MES pH 6.5, 100-150 mM triammonium citrate, 10-12\% (w/v) PEG 20000, and 0.5 M NDSB-195 (Hampton, US)] and subsequently improved by iterative micro-seeding. The crystals were soaked for $1-2 \mathrm{~min}$ in $1 \mathrm{M}$ $\mathrm{NaBr}$ before flash freezing in $15 \%$ (v/v) glycerol. Diffraction datasets were collected on the I04-1 beamline at Diamond Light Source (Didcot, UK) using a Pilatus 6M detector and $0.916 \AA$ beam wavelength. Datasets from multiple crystals were integrated, scaled, and merged using DIALS (42) in the xia2 pipeline (43). Experimental phases and an initial main-chain trace were obtained from SHELX (44). The structure was refined using the CCP4 package (45) and Phenix (46), with iterative rebuilding in Coot.

\section{Structural bioinformatics}


Details of the surface area and r.m.s.d. calculations are found in Supplementary Information.

Database searches against homologs and motifs were carried out using the PlasmoDB database (47), ScanProsite (48), MyHits (49), and PATTINPROT (50). Model of PbCPa ${ }^{\Delta \mathrm{C} 20}$ capped PfActI filament was prepared by aligning 5 protomers of PfActI filament [PDB ID: 5OGW (51)] and $P b C P \alpha \alpha^{\Delta \mathrm{C} 20}$ to the CapZ $\alpha \beta$ capped barbed end of the Arp1 filament in dynactin [PDB ID: 6F1T (28)] using TM-align (52). Gaps in the structure of $P b C P \alpha \alpha^{\Delta \mathrm{C} 20}$ were modeled in using SWISSMODEL. Relative orientation of $P b C P \alpha \alpha^{\Delta \mathrm{C} 20}$ was refined with RosettaDock (53), while residues involved in the interface were energy minimized with UCSF Chimera. Electrostatic potential surfaces were calculated using APBS (54). EMBOSS Matcher (55) was used for local sequence alignments and ESPript (56) for their visualization. 21 non-redundant Plasmodium CPa sequences from PlasmoDB were analyzed, with conservation score subsequently mapped on the structure of $P b \mathrm{CP} \alpha \alpha^{\Delta \mathrm{C} 20}$ using ConSurf (57). CapZ $\alpha \beta$ was prepared similarly, using automatically retrieved homolog sequences.

\section{Actin-CP interaction assays}

Fluorescence-based polymerization assays were carried out in triplicate and analyzed as previously described (27) with minor modifications. $\mathrm{Cc}_{\text {app }}$ was determined using a dilution series prepared from $10 \mu \mathrm{M}$ actin polymerized together with either $10 \mu \mathrm{M} \mathrm{CP}$ or $0.4 \mu \mathrm{M}$ gelsolin for PfActI, and $0.4 \mu \mathrm{M} \mathrm{CP}$ for $\alpha$-actin, respectively. EGTA was omitted from the buffers for the samples with gelsolin. In the polymerization assays, the total actin concentration was kept at $4 \mu \mathrm{M} .2 \mu \mathrm{M} \alpha$-actin, or $0.5 \mu \mathrm{M}$ PfActI filaments ( $2 \mu \mathrm{M}$ in the CapZ $\alpha \beta$ assay) were used as nuclei in preseeded polymerization assays. In the depolymerization assays, CPs were incubated with 2-5 $\mu \mathrm{M}$ actin filaments for $5 \mathrm{~min}$ at $20^{\circ} \mathrm{C}$ prior to 100 -fold dilution. The polymerization data were despiked before averaging. No pointed end cappers were used in the assays to limit subunit exchange to the barbed end.

\section{Acknowledgements}

We thank Dr. Herwig Schüler for providing the parasite CP cDNA constructs and Dr. Shuichi Takeda for the CapZ $\alpha \beta$ plasmid. The use of the facilities and expertise of the Biocenter Oulu Structural Biology core facility is gratefully acknowledged. We also thank the excellent beamline 
support at Diamond Light Source (B21 and I04-1) and the crystallographic community for their helpful comments on the structure of $P b C P \alpha \alpha^{\Delta \mathrm{C} 20}$. This work was supported by the Academy of

Finland, the Sigrid Juselius Foundation, and the Norwegian Research Council. The atomic coordinates and structure factors of $P b \mathrm{CP} \alpha \alpha^{\Delta \mathrm{C} 20}$ have been deposited in the Protein Data Bank, https://www.ebi.ac.uk/pdbe/ (PDB ID: 7A0H).

\section{References}

1. T. D. Pollard, Actin and Actin-Binding Proteins. Cold Spring Harb. Perspect. Biol. 8 (2016).

2. J. A. Cooper, D. Sept, New Insights into Mechanism and Regulation of Actin Capping Protein. Int. Rev. Cell Mol. Biol. 267, 183-206 (2008).

3. M. A. Wear, A. Yamashita, K. Kim, Y. Maéda, J. A. Cooper, How capping protein binds the barbed end of the actin filament. Curr. Biol. 13, 1531-1537 (2003).

4. M. R. Mejillano, et al., Lamellipodial versus filopodial mode of the actin nanomachinery: Pivotal role of the filament barbed end. Cell 118, 363-373 (2004).

5. L. Urnavicius, et al., The structure of the dynactin complex and its interaction with dynein. Science (80-. ). 347, 1441-1446 (2015).

6. D. A. Schafer, J. A. Waddle, J. A. Cooper, Localization of CapZ during myofibrillogenesis in cultured chicken muscle. Cell Motil. Cytoskeleton 25, 317-335 (1993).

7. K. Mukherjee, et al., Actin capping protein CAPZB regulates cell morphology, differentiation, and neural crest migration in craniofacial morphogenesis. Hum. Mol. Genet. 25, 1255-1270 (2016).

8. T. P. Loisel, R. Boujemaa, D. Pantaloni, M. F. Cartier, Reconstitution of actin-based motility of Listeria and Shigella using pure proteins. Nature 401, 613-616 (1999).

9. C. Hug, et al., Capping protein levels influence actin assembly and cell motility in dictyostelium. Cell 81, 591-600 (1995).

10. M. J. DiNubile, L. Cassimeris, M. Joyce, S. H. Zigmond, Actin filament barbed-end capping activity in neutrophil lysates: The role of capping protein- 32 . Mol. Biol. Cell 6, 1659-1671 (1995).

11. D. Pantaloni, C. Le Clainche, M. F. Carlier, Mechanism of actin-based motility. Science (80-. ). 292, 1502-1506 (2001).

12. S. H. Zigmond, Beginning and Ending an Actin Filament: Control at the Barbed End. Curr. Top. Dev. Biol. 63, 145-188 (2004).

13. A. Yamashita, K. Maeda, Y. Maéda, Crystal structure of CapZ: Structural basis for actin filament barbed end capping. EMBO J. 22, 1529-1538 (2003).

14. C. Eckert, A. Goretzki, M. Faberova, M. Kollmar, Conservation and divergence between cytoplasmic and muscle-specific actin capping proteins: Insights from the crystal structure of cytoplasmic Cap32/34 from Dictyostelium discoideum. BMC Struct. Biol. 12 (2012).

15. A. Narita, S. Takeda, A. Yamashita, Y. Maéda, Structural basis of actin filament capping at the barbed-end: A cryo-electron microscopy study. EMBO J. 25, 5626-5633 (2006).

16. T. Kim, J. A. Cooper, D. Sept, The Interaction of Capping Protein with the Barbed End of the Actin Filament. J. Mol. Biol. 404, 794-802 (2010).

17. E. P. Kumpula, I. Kursula, Towards a molecular understanding of the apicomplexan actin motor: On a road to novel targets for malaria remedies? Acta Crystallogr. Sect. FStructural Biol. Commun. 71, 500-513 (2015).

18. H. Schüler, K. Matuschewski, Regulation of apicomplexan microfilament dynamics by a minimal set of actin-binding proteins. Traffic 7, 1433-1439 (2006).

19. M. Ganter, H. Schüler, K. Matuschewski, Vital role for the Plasmodium actin capping protein (CP) beta-subunit in motility of malaria sporozoites. Mol. Microbiol. 74, 1356-1367 (2009).

20. M. C. Hart, Y. O. Korshunova, J. A. Cooper, Vertebrates have conserved capping protein $\alpha$ isoforms with specific expression patterns. Cell Motil. Cytoskeleton 38, 120-132 (1997).

21. U. Haus, H. Hartmann, P. Trommler, A. A. Noegel, M. Schleicher, F-Actin capping by cap32 
34 requires heterodimeric conformation and can be inhibited with PIP2. Biochem. Biophys. Res. Commun. 181, 833-839 (1991).

22. I. Tardieux, I. Baines, M. Mossakowska, G. E. Ward, Actin-binding proteins of invasive malaria parasites and the regulation of actin polymerization by a complex of $32 / 34-\mathrm{kDa}$ proteins associated with heat shock protein 70kDa. Mol. Biochem. Parasitol. 93, 295-308 (1998).

23. M. Ganter, Z. Rizopoulos, H. Schüler, K. Matuschewski, Pivotal and distinct role for Plasmodium actin capping protein alpha during blood infection of the malaria parasite. Mol. Microbiol. 96, 84-94 (2015).

24. Á. Á. Bendes, M. Chatterjee, B. Götte, P. Kursula, I. Kursula, Functional homo- and heterodimeric actin capping proteins from the malaria parasite. Biochem. Biophys. Res. Commun. (2020) https:/doi.org/10.1016/j.bbrc.2020.02.119.

25. L. S. Swapna, K. Srikeerthana, N. Srinivasan, Extent of structural asymmetry in homodimeric proteins: Prevalence and relevance. PLoS One 7 (2012).

26. J. Vahokoski, et al., Structural Differences Explain Diverse Functions of Plasmodium Actins. PLoS Pathog. 10 (2014).

27. E. P. Kumpula, et al., Apicomplexan actin polymerization depends on nucleation. Sci. Rep. 7, 1-10 (2017).

28. L. Urnavicius, et al., Cryo-EM shows how dynactin recruits two dyneins for faster movement. Nature 554, 202-206 (2018).

29. H. Lu, P. M. Fagnant, K. M. Trybus, Unusual dynamics of the divergent malaria parasite PfAct1 actin filament. Proc. Natl. Acad. Sci. U. S. A. 116, 20418-20427 (2019).

30. M. Barzik, et al., Ena/VASP proteins enhance actin polymerization in the presence of barbed end capping proteins. J. Biol. Chem. 280, 28653-28662 (2005).

31. K. Kim, et al., Structure/function analysis of the interaction of phosphatidylinositol 4,5bisphosphate with actin-capping protein: Implications for how capping protein binds the actin filament. J. Biol. Chem. 282, 5871-5879 (2007).

32. I. Siden-Kiamos, H. Schüler, D. Liakopoulos, C. Louis, Arp1, an actin-related protein, in Plasmodium berghei. Mol. Biochem. Parasitol. 173, 88-96 (2010).

33. A. H. Crevenna, et al., Electrostatics control actin filament nucleation and elongation kinetics. J. Biol. Chem. 288, 12102-12113 (2013).

34. M. B. Heintzelman, Cellular and Molecular Mechanics of Gliding Locomotion in Eukaryotes. Int. Rev. Cytol. 251, 79-129 (2006).

35. D. I. Svergun, Restoring low resolution structure of biological macromolecules from solution scattering using simulated annealing. Biophys. J. 76, 2879-2886 (1999).

36. A. Waterhouse, et al., SWISS-MODEL: Homology modelling of protein structures and complexes. Nucleic Acids Res. 46, W296-W303 (2018).

37. G. Tria, H. D. T. Mertens, M. Kachala, D. I. Svergun, Advanced ensemble modelling of flexible macromolecules using X-ray solution scattering. IUCrJ 2, 207-217 (2015).

38. J. Yang, Y. Zhang, I-TASSER server: New development for protein structure and function predictions. Nucleic Acids Res. 43, W174-W181 (2015).

39. P. Emsley, B. Lohkamp, W. G. Scott, K. Cowtan, Features and development of Coot. Acta Crystallogr. Sect. D Biol. Crystallogr. 66, 486-501 (2010).

40. E. F. Pettersen, et al., UCSF Chimera - A visualization system for exploratory research and analysis. J. Comput. Chem. 25, 1605-1612 (2004).

41. A. Panjkovich, D. I. Svergun, Deciphering conformational transitions of proteins by small angle X-ray scattering and normal mode analysis. Phys. Chem. Chem. Phys. 18, 5707-5719 (2016).

42. G. Winter, et al., DIALS: Implementation and evaluation of a new integration package. Acta Crystallogr. Sect. D Struct. Biol. 74, 85-97 (2018).

43. G. Winter, xia2: an expert system for macromolecular crystallography data reduction. $J$. Appl. Crystallogr. 43, 186-190 (2010).

44. G. M. Sheldrick, Experimental phasing with SHELXC/D/E: Combining chain tracing with density modification. Acta Crystallogr. Sect. D Biol. Crystallogr. 66, 479-485 (2010).

45. M. D. Winn, et al., Overview of the CCP4 suite and current developments. Acta Crystallogr. Sect. D Biol. Crystallogr. (2011) https:/doi.org/10.1107/S0907444910045749.

46. P. D. Adams, et al., PHENIX: A comprehensive Python-based system for macromolecular structure solution. Acta Crystallogr. Sect. D Biol. Crystallogr. 66, 213-221 (2010). 
47. C. Aurrecoechea, et al., PlasmoDB: A functional genomic database for malaria parasites. Nucleic Acids Res. 37 (2009).

48. E. de Castro, et al., ScanProsite: Detection of PROSITE signature matches and ProRuleassociated functional and structural residues in proteins. Nucleic Acids Res. (2006) https:/doi.org/10.1093/nar/gkl124.

49. M. Pagni, et al., MyHits: Improvements to an interactive resource for analyzing protein sequences. Nucleic Acids Res. (2007) https:/doi.org/10.1093/nar/gkm352.

50. C. Combet, C. Blanchet, C. Geourjon, G. Deléage, NPS@: Network protein sequence analysis. Trends Biochem. Sci. (2000) https:/doi.org/10.1016/S0968-0004(99)01540-6.

51. S. Pospich, et al., Near-atomic structure of jasplakinolide-stabilized malaria parasite F-actin reveals the structural basis of filament instability. Proc. Natl. Acad. Sci. U. S. A. 114, 1063610641 (2017).

52. Y. Zhang, J. Skolnick, TM-align: A protein structure alignment algorithm based on the TMscore. Nucleic Acids Res. 33, 2302-2309 (2005).

53. R. Moretti, S. Lyskov, R. Das, J. Meiler, J. J. Gray, Web-accessible molecular modeling with Rosetta: The Rosetta Online Server that Includes Everyone (ROSIE). Protein Sci. (2018) https:/doi.org/10.1002/pro.3313.

54. E. Jurrus, et al., Improvements to the APBS biomolecular solvation software suite. Protein Sci. 27, 112-128 (2018).

55. F. Madeira, et al., The EMBL-EBI search and sequence analysis tools APIs in 2019. Nucleic Acids Res. (2019) https:/doi.org/10.1093/nar/gkz268.

56. X. Robert, P. Gouet, Deciphering key features in protein structures with the new ENDscript server. Nucleic Acids Res. 42 (2014).

57. H. Ashkenazy, et al., ConSurf 2016: an improved methodology to estimate and visualize evolutionary conservation in macromolecules. Nucleic Acids Res. (2016)

https:/doi.org/10.1093/nar/gkw408. 


\section{Figure legends}

Fig. 1. $\mathrm{P} b \mathrm{CP}$ homo- and heterodimers have a canonical shape in solution. (A) Experimental scattering curves of $P b C P \alpha \beta, P b C P \alpha \alpha$, and $P b C P \alpha \alpha^{\Delta C 20}$ (red, green, and blue open circles, respectively) and the respective fits of DAMMIN (white dashed lines) and SREFLEX (black line) models to the data. The $\chi^{2}$ values of the different models to the data are indicated. Weighted residuals of the SREFLEX model fits are denoted in the lower graph (lines colored respectively), where $\Delta / \square=\left[\square_{\square \square \square}(\square)-\square \square_{\square \square \square}(\square)\right] / \square(\square)$. (B) Dimensionless Kratky plot (colored similarly to panel A). (C) Real-space distance distribution plot (colored similarly to panel A). (D) Cartoon models of $\mathrm{PbCPs}$ (colored as in panel A, with chain A in lighter color) and the corresponding SAXS envelopes as surface representations. The tentacle domains of each structure are colored black. Experimental data for $\mathrm{PbCP} \alpha \alpha^{\Delta \mathrm{C} 20}$ for comparison were used from a previous publication (24).

Fig. 2. Crystal structure and domain arrangement of $P b C P \alpha \alpha^{\Delta \mathrm{C} 20}$. (A) Crystal structure of $P b C P a \alpha^{\Delta \mathrm{C} 20}$. (B) Superposition of the $P b C P \alpha_{1}{ }^{\Delta \mathrm{C} 20}$ and $P b C P \alpha_{2}{ }^{\Delta \mathrm{C} 20}$ subunits. The rainbow color scale represents the r.m.s.d. between the subunits. Residues excluded from the alignment are colored black. (C) Superposition of $P b C P \alpha_{1}{ }^{\Delta \mathrm{C} 20}$ to $\mathrm{CapZ} \alpha$ (left) and $P b C P \alpha_{2}{ }^{\Delta \mathrm{C} 20}$ to CapZ $\beta$ (right). The domains of $P b C P \alpha \alpha^{\Delta \mathrm{C} 20}$ are colored as in panel A. CapZ $\alpha \beta$ [PDB ID: 1IZN (13)] is shown in light gray.

Fig. 3. $P b C P s$ do not affect the critical concentration of actin polymerization. (A) PfActI filaments (open black squares) capped by $P b C P \alpha \beta, P b C P \alpha \alpha, P b C P \alpha \alpha^{\Delta \mathrm{C} 20}$, and CapZ $\alpha \beta$ (open red circles, green diamonds, blue triangles, and purple stars, respectively). The gray shading indicates the range of determined $\mathrm{Cc}_{\text {app }}$ from two-line fits (respectively colored lines). (B) Cc plot of PfActI filaments (open black squares) with gelsolin (open gray pentagon) under $\mathrm{Ca}^{2+}$ conditions. (C) $\alpha$-actin filaments (open black squares) capped by $P b \mathrm{CP} \alpha \beta, P b \mathrm{CP} \alpha \alpha, P b C P \alpha \alpha^{\Delta \mathrm{C} 20}$, and $\mathrm{CapZ} \alpha \beta$ (as in panel A). Errors represent SD $(n=3)$. RFU = relative fluorescent unit, normalized to the lowest concentration. 
Fig. 4. Atypical regulation of polymerization kinetics by PbCPs. (A) Polymerization curves of $P f A c t I$ in the absence and presence of increasing concentrations of $P b C P \alpha \beta, P b C P \alpha \alpha, P b C P \alpha \alpha^{\Delta C 20}$, and CapZ $\alpha \beta$. (B) Polymerization of PfActI on capped, preformed homologous filaments in the absence and presence of increasing concentrations of $P b \mathrm{CP} \alpha \beta, P b \mathrm{CP} \alpha \alpha, P b C P \alpha \alpha^{\Delta \mathrm{C} 20}$, and $\mathrm{CapZ} \alpha \beta$. (C and D) Non-seeded and seeded polymerization curves of $\alpha$-actin with the different CPs, as in panels A and B. RFU = relative fluorescent unit.

Fig. 5. $\mathrm{PbCPs}$ facilitate filament depolymerization. (A) Dilution-induced depolymerization of PfActI filaments (black line) with various concentrations of $P b \mathrm{CP} \alpha \beta, P b \mathrm{CP} \alpha \alpha, P b \mathrm{CP} \alpha \alpha^{\Delta \mathrm{C} 20}$, and CapZ $\alpha \beta$. (B) Depolymerization of $P b C P \alpha \beta, P b C P \alpha \alpha, P b C P \alpha \alpha^{\Delta C 20}$, and CapZ $\alpha \beta$ capped $\alpha$-actin filaments (black line). RFU = relative fluorescent unit.

Fig. 6. Atypical structural properties of $P f A c t I$ and $P b C P \alpha \alpha^{\Delta \mathrm{C} 20}$ suggest new barbed end binding modes. (A) Model of PfActI filament [green to beige colored surfaces, PDB ID: 5OGW (51)] capped by $P b C P \alpha \alpha^{\Delta \mathrm{C} 20}$ (blue cartoon) in a canonical arrangement. (B) Electrostatic potential surface of the last (b) and penultimate (b-1) protomer of PfActI (left) and Arp1 [right, PDB ID: 6F1T (28)] filament barbed ends. (C) Electrostatic potential surface of $P b C \mathrm{P} \alpha \alpha^{\Delta \mathrm{C} 20}$ (left) and CapZ $\alpha \beta$ [right, PDB ID: 6F1T (28)]. (D) The hydrophobic pocket of the last subunit of PfActI (left) and Arp1 (right) filament, represented as green surface. The Plasmodium-specific insert, cap sheets, and cap helices of $P b C P \alpha \alpha^{\Delta C 20}$ (left) are colored purple, red, and teal, respectively. The CapZ $\alpha \beta$ (right) $\alpha$ and $\beta$-subunits are shown in light and dark gray, respectively. Sequence alignment (bottom) of the Plasmodium insert (PbCPa_ins) with the $\beta$-tentacles of P. berghei (PbCPb_C: A0A509AQN8) and S. scrofa CP (SsCPb_C: A9XFX6), grouped and colored respective to a Risler matrix, using the ESPript convention. (E) Structure of $P b C P \alpha \alpha^{\Delta \mathrm{C} 20}$ (left) and CapZ $\alpha \beta$ (right) with the surface colored by ConSurf residue conservation scores from low (cyan) to high (magenta). 
Table I. Data collection and refinement statistics

\begin{tabular}{|c|c|}
\hline Data collection & $P b C P \alpha \alpha^{\Delta C 20}$ \\
\hline Wavelength $(\AA)$ & 0.916 \\
\hline Resolution range $(\AA)$ & $79.65-2.22(2.30-2.22)^{*}$ \\
\hline Space group & C 121 \\
\hline \multicolumn{2}{|l|}{ Cell dimensions } \\
\hline $\mathbf{a}, \mathbf{b}, \mathbf{c}(\AA)$ & $164.49,35.17,115.44$ \\
\hline $\boldsymbol{\alpha}, \boldsymbol{\beta}, \boldsymbol{\gamma}\left({ }^{\circ}\right)$ & $90.00,104.44,90.00$ \\
\hline No. of reflections & $2516659(51165)$ \\
\hline Unique $^{\dagger}$ & 61855 (6109) \\
\hline Multiplicity & $40.7(8.4)$ \\
\hline Completeness (\%) & $99.24(95.57)$ \\
\hline$\langle\mathbf{I} / \sigma(\mathbf{I})\rangle$ & $10.62(0.45)$ \\
\hline Wilson B-factor $\left(\AA^{2}\right)$ & 49.65 \\
\hline $\mathbf{R}_{\text {merge }}$ & $0.260(4.37)$ \\
\hline $\mathbf{R}_{\text {pim }}$ & $0.0395(1.57)$ \\
\hline $\mathrm{CC}_{1 / 2}$ & $0.999(0.29)$ \\
\hline \multicolumn{2}{|l|}{ Refinement } \\
\hline Resolution range $(\AA)$ & $74.20-2.22(2.25-2.22)$ \\
\hline No. of reflections & $61419(2419)$ \\
\hline $\mathbf{R}_{\text {work }}$ & $0.207(0.420)$ \\
\hline $\mathbf{R}_{\text {free }}{ }^{\$}$ & $0.257(0.428)$ \\
\hline \multicolumn{2}{|l|}{ No. of atoms } \\
\hline Protein & 4432 \\
\hline Ligand/ion & 61 \\
\hline Solvent & 149 \\
\hline \multicolumn{2}{|l|}{ r.m.s.d. } \\
\hline Bond lengths $(\AA)$ & 0.016 \\
\hline Bond angles $\left(^{\circ}\right)$ & 1.47 \\
\hline \multicolumn{2}{|l|}{ B-factors $\left(\AA^{2}\right)$} \\
\hline Protein & 107.4 \\
\hline Ligand/ion & 93.0 \\
\hline Solvent & 64.6 \\
\hline \multicolumn{2}{|c|}{ Ramachandran plot (\%) } \\
\hline Favored & 96.42 \\
\hline Outliers & 0.00 \\
\hline Rotamer outliers (\%) & 0.59 \\
\hline
\end{tabular}


$\mathbf{A}$

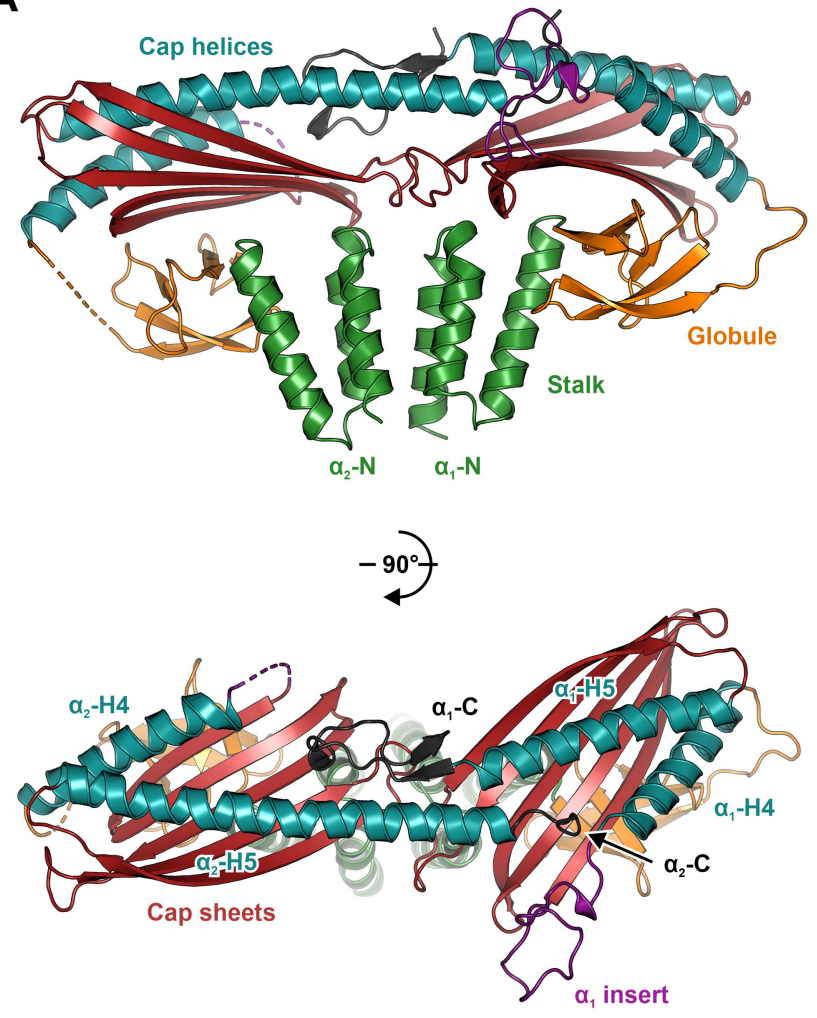

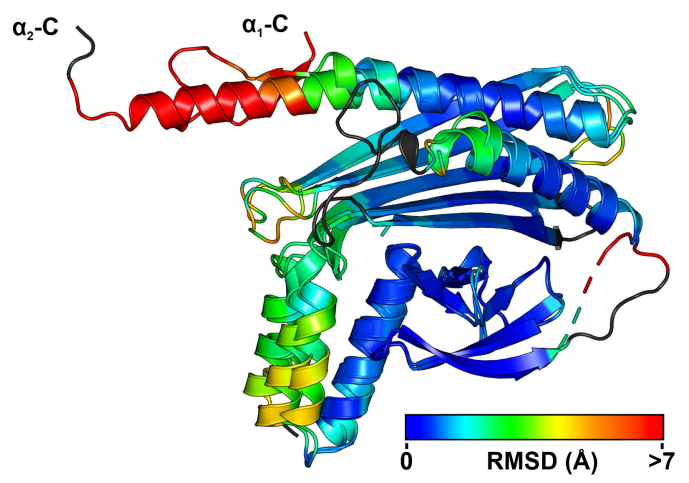

C

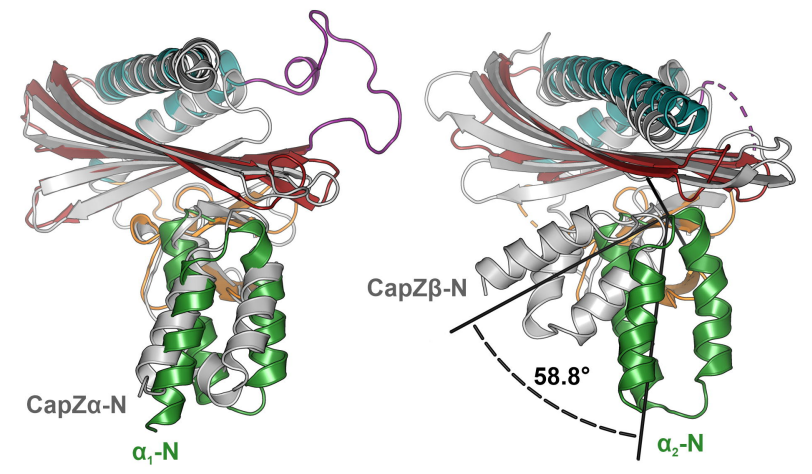


A
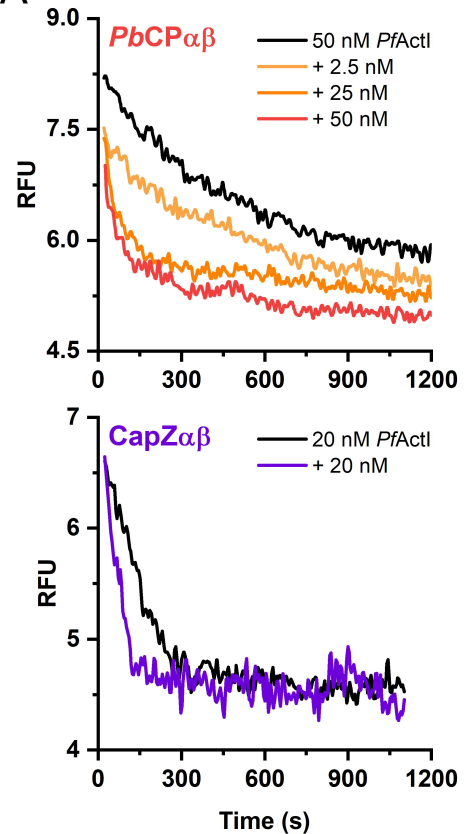
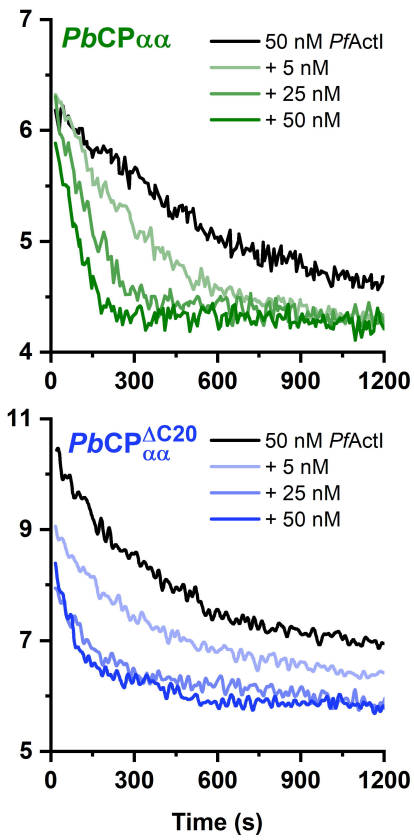
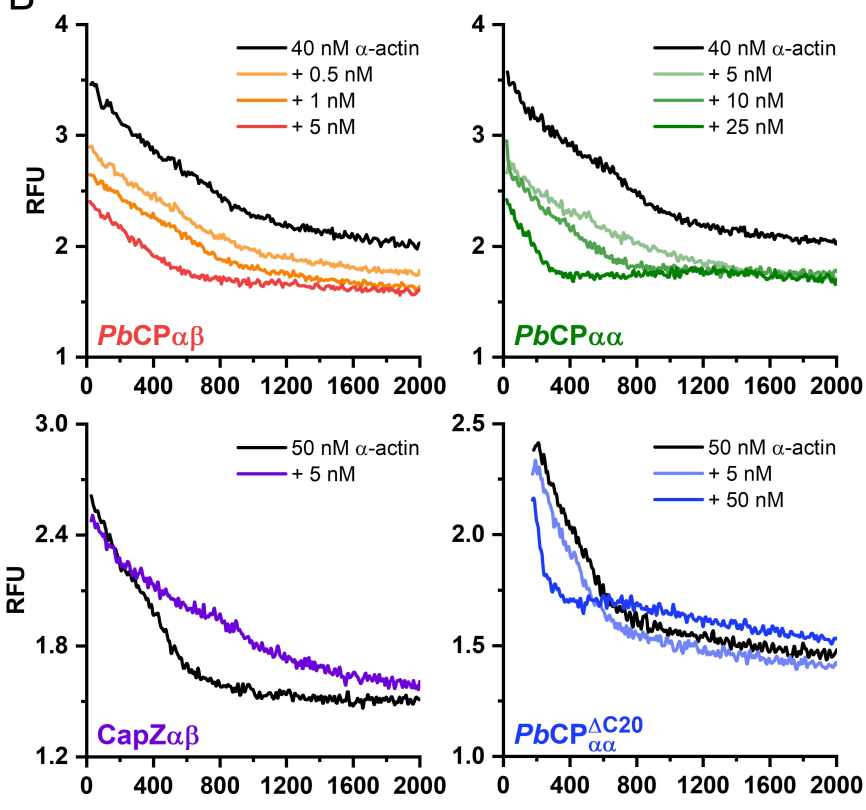

Time (s)

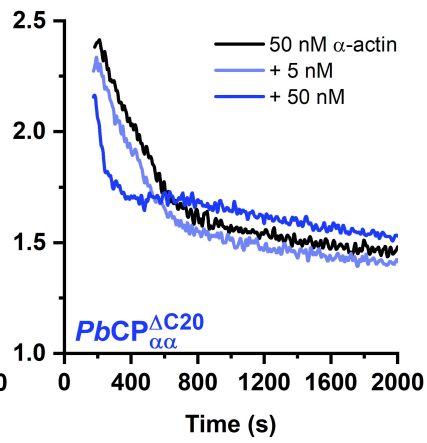




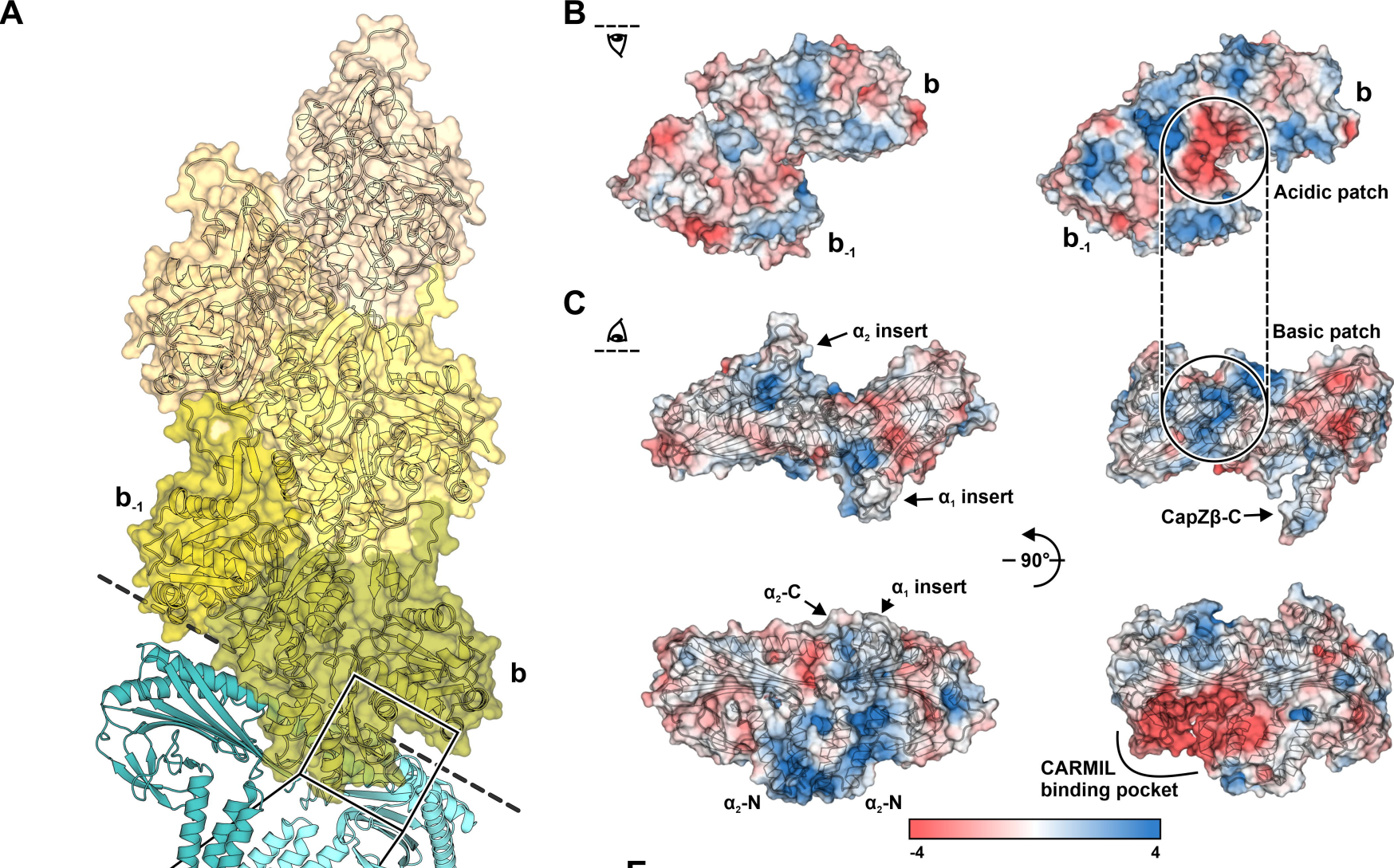

B

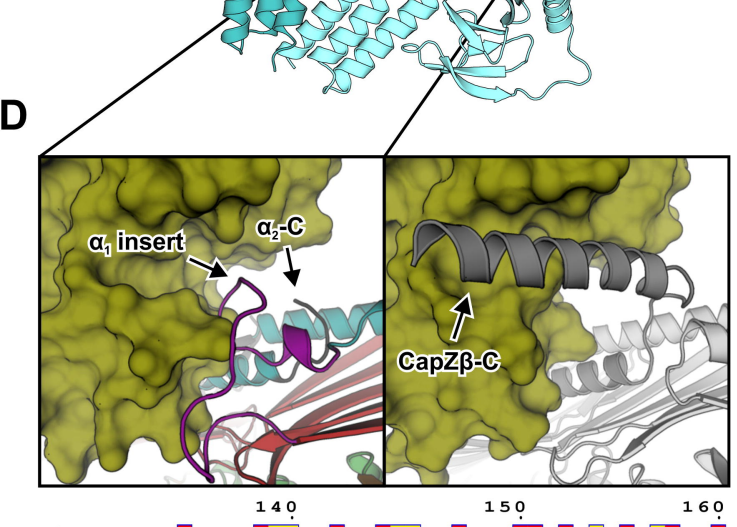

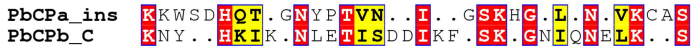
$140 \quad 150$

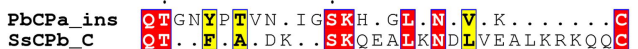

E
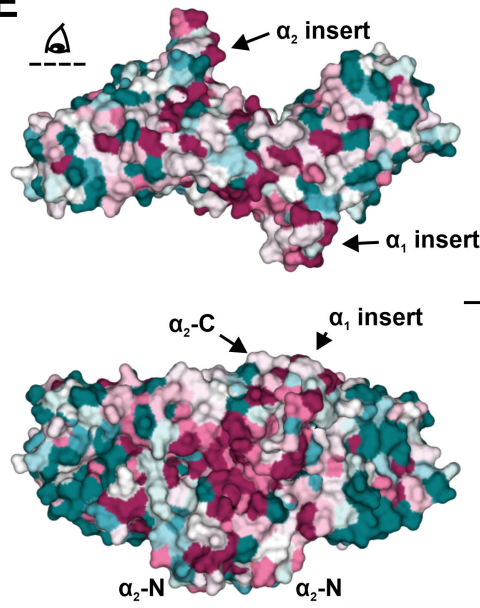

Basic patch
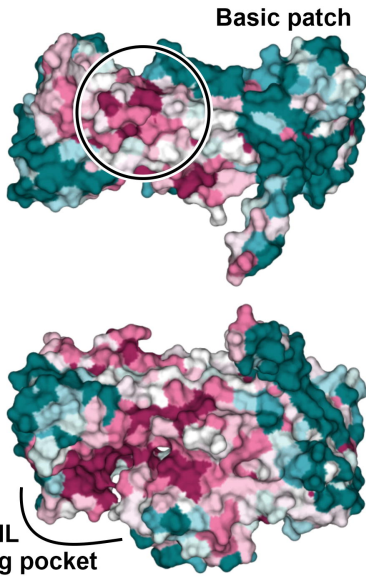
binding pocket

\footnotetext{
$-90^{\circ}$
}

Conserved 